\title{
Actitud hacia la estadística por parte de los estudiantes de Medición Psicológica de la UNAH, 2015
}

Miguel Landa Blanco ${ }^{1}$

\section{RESUMEN}

La presente investigación tuvo como objetivo principal conocer la actitud hacia la estadística por parte de los estudiantes de las clases de Medición Psicológica, de la UNAH, durante el primer período académico de 2015. El abordaje se realizó con un enfoque cuantitativo de alcance descriptivo. Para ello se tomó una muestra de 316 alumnos a los cuales se les aplicó la escala de actitudes hacia la estadística (Auzmendi, 1992), versión validada por Tejero y Castro (2011).

Los resultados indican que el $16 \%$ de los sujetos manifiestan una actitud negativa hacia la estadística, mientras que el $43 \%$ de los alumnos se muestra indiferente ante ella y el $41 \%$ tiende a tener actitudes favorables hacia tal disciplina.

El análisis por factores indica que existe resistencia por parte del alumno a involucrarse en asignaciones con carga estadística. Igualmente, se reconoce la utilidad de la estadística en la psicología, pero existe renuencia a relacionarse personalmente en tareas relacionadas con tal disciplina. Se encontró que la actitud hacia la estadística no varía en función de la asignatura que cursa el alumno, finalmente se concluye que, dentro de la muestra de estudio, los hombres tienden a mostrar una mejor actitud hacia la estadística que las mujeres.

Palabras claves: actitudes, estadística, psicología, psicometría.

${ }_{1}^{1}$ Profesor investigador de la Carrera de Psicología, Facultad de Ciencias Sociales, UNAH: mlandablanco@gmail.com 


\section{ABSTRACT}

The main objective of this research is to know the student's attitude towards statistics, only those enrolled in the Psychological Measurement classes, in the UNAH, during the first term of 2015. It was quantitative study, with a descriptive approach. The sample consisted of 316 students who were administered the Scale of Attitudes towards Statistics (Auzmendi, 1992), validated by Tejero \& Castro (2011). The results indicate that $16 \%$ of the students have a negative attitude towards statistics, while $43 \%$ of the sample showed indifference towards it, on the other hand, $41 \%$ of the students reported favorable attitudes.

A detailed analysis indicates that students consider a burden to be involved in statistical assignments, this includes participation in additional courses and a low level of satisfaction and stimulation associated with such work. Moreover, they recognize the usefulness of statistics in psychology, but are reluctant to become personally involved in related tasks. There is no variation in attitude towards statistics regardless of the subject attended by the student. Finally it is concluded that, within the sample study, men tend to have a better attitude toward statistics than women,

Keywords: attitudes, statistics, psychology, psychometrics. 


\section{INTRODUCCIÓN}

A lo largo de la evolución histórica de la psicología como ciencia se fueron implementando diversas técnicas que procuraban no solo capturar las subjetividades humanas, sino además brindar una medida objetiva de las características psicográficas. Para ello fue necesario implementar abordajes positivistas que permitieran la cuantificación de tales variables. Las pruebas son instrumentos o técnicas de medición útiles para describir y predecir comportamientos (Kaplan y Saccuzzo, 2006). Dichas mediciones incluyen dimensiones relacionadas con la inteligencia, el desarrollo psiconeurológico, personalidad, intereses vocacionales, aptitudes, etc.

El diseño e interpretación de tales test psicológicos requieren de un fuerte componente estadístico, el cual es fundamental para la toma de decisiones en las diversas áreas de aplicación de la psicología. Las pruebas psicométricas comúnmente basan su estructura sobre medidas de tendencia central, percentiles, medidas de dispersión, curva normal, intervalos de confianza y la estadística inherente a la confiabilidad y validez de los test psicológicos.

El plan de estudios de la Carrera de Psicología de la Universidad Nacional Autónoma de Honduras (UNAH) contempla cuatro clases asignadas a la medición psicológica. Dicho bloque tiene como requisito la asignatura de Métodos de investigación psicológica II, la cual basa su contenido en principios de estadística descriptiva e inferencial. Consecuentemente, se asume que el alumno es capaz de aplicar dichos conocimientos en el ámbito psicométrico.

La actitud es una construcción dinámica-evaluativa hacia determinado estímulo, tal elaboración conjuga aspectos afectivos, cognitivos y conductuales. Por lo tanto, son ampliamente influyentes en la ejecución funcional del sujeto, la misma puede tener un impacto constructivo o destructivo en la realización de una tarea. Es por ello que las actitudes intervienen sobre las conductas finales del sujeto, variando en función a su orientación e intensidad (Baron y Byrne, 2005).

Los alumnos de pregrado tienden a percibir la estadística como uno de los mayores desafíos de su vida estudiantil (Coetzee y Van Der Merwe, 2010), por consiguiente, esto plantea una situación problemática entre las exigencias académicas profesionales y las actitudes personales. La resolución positiva de dicha fricción resultaría en estudiantes capaces y dispuestos a aplicar la estadística a las labores psicométricas; mientras que una actitud negativa limitaría la adecuación de tal integración. 
En consecuencia, se plantea el objetivo general de conocer la actitud hacia la estadística de los estudiantes cursando las clases de Medición Psicológica, de la UNAH-CU, durante el primer período académico de 2015. Para ello es necesario describir la orientación actitudinal hacia la estadística, así como los factores psicológicos que influyen en ella; para luego contrastar la actitud hacia la estadística en función del género y la asignatura de Medición Psicológica que cursa la población de estudio.

Esto permitirá determinar las fortalezas y puntos de mejoras que deben ser atendidos para poder fomentar una actitud favorable hacia la estadística en los estudiantes de psicología. Asimismo, será posible establecer tales estrategias psicopedagógicas basándose en mediciones objetivas, congruentes con la realidad. De igual manera, beneficiará a estudiantes y profesores de la carrera de psicología, extensivamente será posible ofrecer un mejor servicio para aquellos sujetos que se sometan a evaluaciones psicométricas, con lo cual se podrá aportar al desarrollo de la psicología en sus diferentes campos de acción.

\section{METODOLOGÍA}

\section{Diseño}

La presente investigación se enmarca en el paradigma positivista, el cual plantea la existencia de una realidad objetiva (Sandoval, 1996). Para lograr tal conocimiento se realizó un abordaje basado en el enfoque cuantitativo, ya que se estudió el fenómeno de manera numérica, con la utilización instrumentos que permitieron una medición del mismo. El método usado fue el deductivo, ya que utilizó las particularidades para inferir generalidades acerca del objeto de estudio.

El alcance es predominantemente descriptivo, ya que se limita a caracterizar la actitud de los estudiantes hacia la estadística. El tipo de investigación es de corte transversal con un diseño no experimental, lo que indica que no existió manipulación de variables.

\section{Población}

La presente investigación tomó como población de estudio a todos los alumnos matriculados en las clases de Medición Psicológica I, Medición Psicológica II, Medición 
Psicológica III y Medición Psicológica IV. Según los datos obtenidos, tal universo consta de 490 sujetos, dicha información incluye el conteo de alumnos que se retiraron de la asignatura, pero no cancelaron la misma, por lo que el verdadero conteo poblacional es inferior a tal cifra.

Se calculó el tamaño de la muestra utilizando el programa Epi Info 7, con el cual a un nivel de confianza del $99 \%$ ( $z=2.58)$, con una frecuencia esperada del $50 \%$, un límite de confianza del $5 \%$, se obtuvo una muestra de 312 individuos. La selección de dichos casos fue de manera no probabilística, de tipo incidental. Finalmente, se encuestaron a 316 sujetos, lo cual equivale al $64 \%$ de la población de estudio. De dicha muestra, el $80.4 \%$ de los individuos eran del género femenino (80.4\%) y el $19.6 \%$ masculino. La edad promedio de los participantes fue de 25 años de edad.

\section{Instrumento}

Para la recolección de datos se utilizó la escala de actitud hacia la estadística elaborada por Auzmendi (1992), versión validada por Tejero y Castro (2011). Dicha escala obedece a un formato de autorreporte tipo Likert, con 5 posibilidades de respuesta. Los factores que componen esta escala revisada son la respuesta fisiológica de calma o no ansiedad ( $\alpha=.87$ ), la predisposición activa y positiva $(\alpha=.83)$ y la percepción de utilidad e importancia ( $\alpha=.76)$.

La escala consta de 12 ítems, a continuación se presenta una muestra de tales reactivos: "la estadística es una de las asignaturas que más temo, el utilizar la estadística es una diversión para mí, considero la estadística como una materia muy necesaria en la carrera". Complementariamente, se recogieron datos demográficos y educativos de los participantes, con el fin de enriquecer el análisis de los resultados. Fue necesario realizar una recodificación inversa para las puntuaciones de ítems con orientación negativa.

\section{ANÁLISIS DE RESULTADOS}

\section{Consideraciones previas}

No hay consenso en cuanto al nivel de medición a la cual pertenecen los datos que ofrece una escala Likert. Para el presente estudio se utilizó un tratamiento de datos de tipo intervalar, similar al manejado por los autores de la versión revisada del 
instrumento aplicado (Tejero y Castro, 2011). Se considera apropiado el uso de estadísticos descriptivos como la media y la desviación estándar, así como el uso de pruebas paramétricas, tales como análisis de varianza y correlaciones de Pearson (Carifio y Perla, 2008). Complementariamente, se ha encontrado que el tipo de análisis, paramétrico o no paramétrico, no afecta las conclusiones obtenidas en los resultados de las escalas Likert (Murray, 2013; Sullivan y Artino, 2013); es común que se analicen dichos instrumentos como pertenecientes a la escala de intervalo (Hernández, Fernández y Baptista, 2010).

\section{Descripción general de la actitud hacia la estadística}

El $16 \%$ de los sujetos manifiestan una actitud negativa hacia la estadística (ver gráfico 1). El $43 \%$ se muestra indiferente y el $41 \%$ tiende a tener actitudes favorables hacia la estadística. Por lo tanto, la mayor parte de los alumnos tienen actitudes indiferentes o negativas hacia la estadística.

Gráfico 1. Distribución de los resultados generales de la actitud hacia la estadística

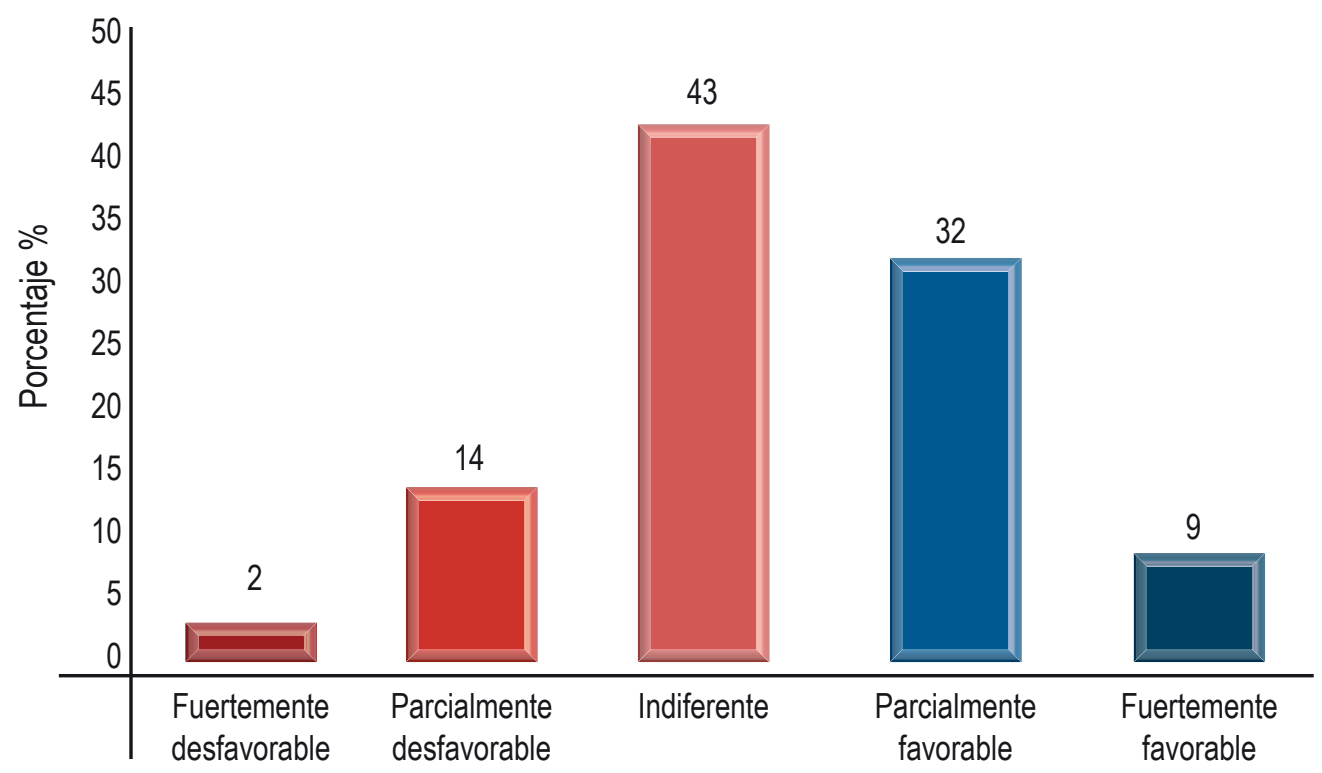

El factor actitudinal que obtuvo el mayor puntaje ( $x=3.5235, s=.81612)$ fue la percepción de utilidad e importancia que los alumnos le otorgan a la estadística. Sin embargo, tal estimación se clasifica como parcialmente favorable, por lo cual únicamente se podría interpretar como una tendencia a valorar el rol de la estadística en la 
vida profesional y académica. Otros estudios han encontrado que otorgar valor a la estadística está positivamente relacionado con las calificaciones de los alumnos, sugiriendo que la metodología de enseñanza debe ofrecer las condiciones necesarias para que el alumno logre distinguir la utilidad de la estadística en las aplicaciones profesionales (Slootmaeckers, 2012).

El segundo factor con mayor puntaje $(x=3.4929, s=.85623)$ corresponde a la respuesta fisiológica de no ansiedad o calma, el cual describe la respuesta de sosiego 0 zozobra del alumno ante la ejecución de labores estadísticas. La puntuación obtenida indica que, en promedio, los encuestados tienen manifestaciones fisiológicas parcialmente favorables ante la estadística. El factor de predisposición activa y positiva obtuvo una puntuación clasificada como indiferente $(x=2.7761, s=.90035)$. Lo cual indica resistencia del alumno a involucrarse en asignaciones con carga estadística, esto incluye la participación en cursos complementarios y el nivel de satisfacción y estimulación que se obtiene al realizar tales labores (ver gráfico 2).

Gráfico 2. Descripción de los resultados disgregados por factores

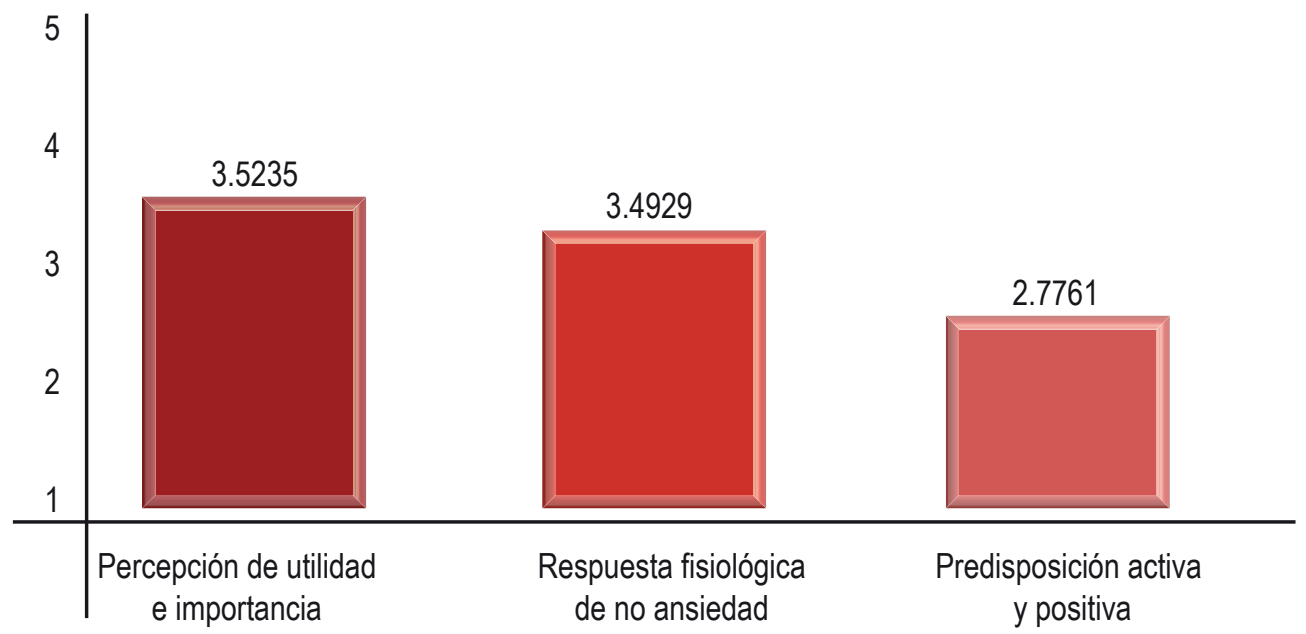

Se realizó una prueba T para muestras dependientes, con el fin de comparar los puntajes entre los tres factores evaluados por la escala aplicada. Los resultados indican que no existe diferencia estadísticamente significativa entre las medias para los factores de percepción de utilidad e importancia ( $\mathrm{x}=3.5235, \mathrm{~s}=.81612)$ y los de respuesta fisiológica de calma o no ansiedad $(x=3.4929, s=.85623) ; t(315)=.579$, $p=.563$. Lo que indica que ambas variables se desarrollan de manera similar. 
También existe diferencia estadísticamente significativa entre los puntajes de percepción de utilidad e importancia $(x=3.5235, s=.81612)$ y los de predisposición activa y positiva $(x=2.7761, s=.90035) ; t(315)=14.021, p=.000$. De tal forma que los estudiantes encuestados tienden a reconocer, de manera moderada, el valor de la estadística y sus aplicaciones académicas-laborales. Sin embargo, esta apreciación es predominantemente contemplativa, ya que existe una actitud indiferente hacia la participación activa en asignaciones con contenido estadístico. Por lo tanto, se reconoce la utilidad de la estadística en la psicología, pero existe renuencia a involucrarse personalmente en faenas relacionadas a ella.

\section{Actitud hacia la estadística en función a la asignatura cursada}

Se puede observar que los puntajes más bajos para la escala total se encuentran en la clase de Medición Psicológica IV ( $x=3.16, s=.73$ ). Al ejecutar la prueba ANOVA, no se encontraron diferencias estadísticamente significativas $\{F(3,312)=1.007, p=.390\}$ entre la actitud hacia la estadística en función a la clase que cursaban los sujetos.

En teoría, los alumnos con mayor experiencia previa en estadística tienden a reportar actitudes más positivas hacia la misma (Ramírez, Schau y Emmioglu, 2012). Considerando el contenido estadístico de la psicometría, se esperaría que un estudiante cursando la clase de Medición Psicológica IV hubiese tenido mayor contacto con la estadística y, por lo tanto, una mejor actitud hacia ella.

Sin embargo, la presente investigación muestra que la actitud hacia la estadística se mantiene estática a lo largo de la evolución académica del alumno de psicología de la UNAH. Así, un alumno que esté finalizando el bloque de clases de Medición Psicológica ha tenido mayor exposición a la estadística utilizada en la psicometría, pero muestra la misma actitud que un principiante en dicha área. Esto podría indicar que las clases de medición psicológica no hacen suficiente énfasis en el fundamento estadístico de las pruebas psicométricas, sino que se centran en la aplicación e interpretación teórica de los test enseñados.

\section{Actitud hacia la estadística en función al género}

Se encontró que los hombres ( $x=3.52, s=.77$ ) tienden a mostrar una mejor actitud hacia la estadística que las mujeres ( $x=3.20, s=.64)$. Se realizó el análisis de varianza para determinar que dicha diferencia es estadísticamente significativa $\{F(1,314)=11.123, p=.001\}$. Otros estudios han encontrado que los hombres tienden, con moderación, a tener afectos más positivos hacia la estadística, en comparación a 
las mujeres (Coetzee y Van Der Merwe, 2010). Sin embargo, estos resultados son contrarios a los obtenidos por otras investigaciones (García, Fallas y Romero, 2015). Por ejemplo, la prueba de aptitud académica (PAA) aplicada por la UNAH, muestra que los varones tienen puntajes más altos que las mujeres en la PAA de matemática y verbal (Universidad Nacional Autónoma de Honduras, 2014).

La disgregación por factores permite apreciar que el género masculino muestra, en promedio, una mejor actitud que las mujeres en todos los componentes escalares (ver gráfico 3). Se ejecutó ANOVA para conocer si las puntuaciones difieren significativamente entre sí. Al respecto, se encontró que no existe diferencia estadísticamente significativa entre las puntuaciones de hombres y mujeres en cuanto a la percepción de utilidad e importancia $\{F(1,314)=.097, p=.756\}$. Sin embargo, el género masculino obtuvo puntuaciones estadísticamente diferentes, en comparación a las mujeres, para los factores de predisposición activa y positiva $\{F(1,314)=20.954, p=.000\}$ y respuesta fisiológica de calma o no ansiedad $\{F(1,314)=8.031, p=, 005\}$.

Esto indica que, en términos relativos, los hombres tienden a ser más permeables ante la formación y aplicación de la estadística, en comparación a las mujeres. De igual forma, se orientan a afrontar dichas labores con más calma que el género femenino. Se ha reportado que los hombres tienen mayores expectativas de rendimiento, autoconcepto y motivación intrínseca hacia la matemática en comparación a las mujeres (Skaalvik y Skaalvik, 2004).

Gráfico 3. Descripción factorial por género

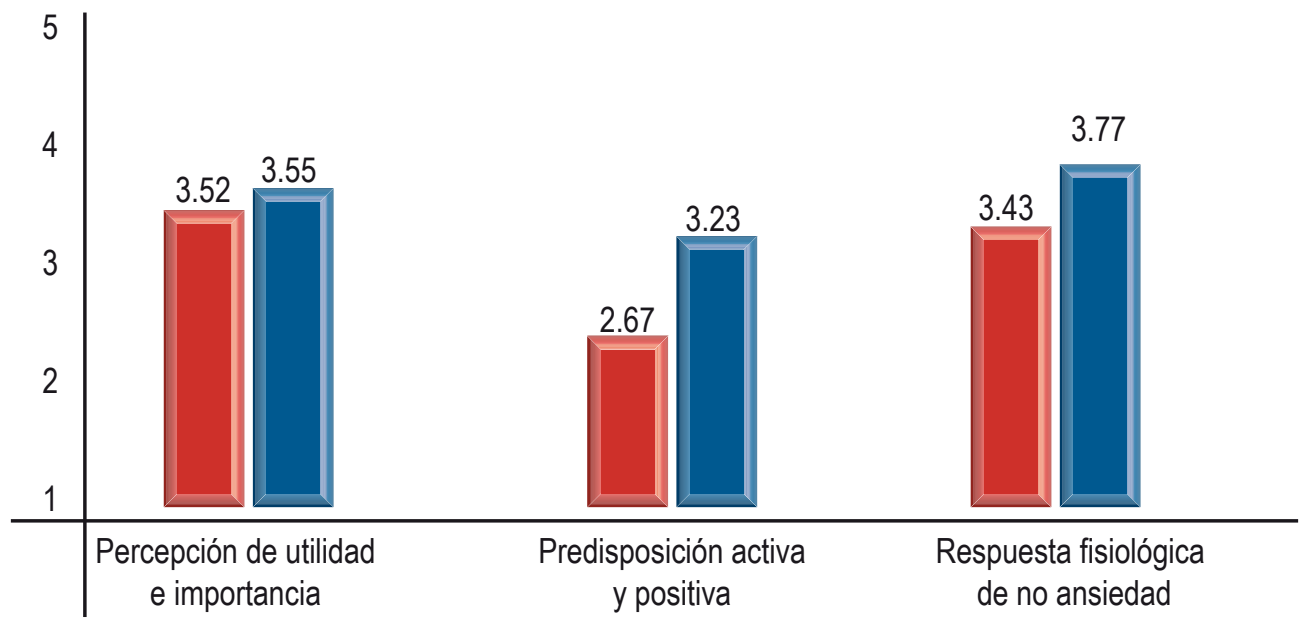




\section{CONCLUSIONES}

1. El $16 \%$ de los sujetos manifiestan una actitud negativa hacia la estadística, mientras que el $43 \%$ de los alumnos se muestra indiferente ante ella y el $41 \%$ tiende a tener actitudes favorables hacia tal disciplina. El factor con la puntuación más alta corresponde a la percepción de utilidad e importancia que los alumnos otorgan a la estadística, dicho promedio se clasifica como parcialmente favorable.

2. El segundo factor con mayor puntaje corresponde a la respuesta fisiológica de no ansiedad o calma. La puntuación obtenida indica que, en general, los encuestados tienen manifestaciones fisiológicas equilibradas ante la estadística.

3. El factor de predisposición activa y positiva obtuvo una puntuación clasificada como parcialmente desfavorable, lo cual indica resistencia del alumno a involucrarse en asignaciones con carga estadística, esto incluye la participación en cursos complementarios y el nivel de satisfacción y estimulación que se obtiene al realizar tales labores.

4. Existe diferencia estadísticamente significativa entre los puntajes de percepción de utilidad e importancia y la predisposición activa/positiva. De esta forma, los estudiantes encuestados tienden a reconocer, de manera moderada, el valor de la estadística y sus aplicaciones académicas-laborales. Sin embargo, esta apreciación es predominantemente contemplativa, ya que existe una actitud indiferente hacia la participación activa en asignaciones con contenido estadístico.

5. Se encontró que la actitud hacia la estadística no varía en función de la asignatura que cursa el alumno, es decir, que un alumno que esté finalizando el bloque de clases de Medición Psicológica muestra la misma actitud que un principiante en la psicometría.

6. Los hombres tienden a mostrar una mejor actitud hacia la estadística que las mujeres, dicho resultado es consistente para todos los componentes factoriales de la escala. 


\section{BIBLIOGRAFÍA}

Auzmendi, E. (1992). Las actitudes hacia la matemática-estadística en las enseñanzas medias y universitaria. España: Bilbao y Mensajero.

Baron, R. y Byrne, D. (2005). Psicología social. Madrid: Pearson Educación.

Carifio, J. y Perla, R. (2008). Revising the 50 year debate around using and misusing Likert scales. Medical Education, 42(12), 1150-1152.

Coetzee, S. y Van Der Merwe, P. (2010). Industrial Psychology Student's Attitudes Towards Statistics. SA Journal of Industrial Psychology, 36(1), 1-8.

García, J.; Fallas, M. y Romero, A. (2015). Las actitudes hacia la estadística del estudiantado de orientación. Revista Electrónica Educare, 19(1), 25-41.

Hernández, R.; Fernández, C. y Baptista, P. (2010). Metodología de la investigación. Perú: McGraw-Hill.

Kaplan, R. y Saccuzzo, D. (2006). Pruebas psicológicas. Méjico: Thomson.

Murray, J. (2013). Likert data: What to use, Parametric or Non-Parametric. International Journal of Business and Social Science, 4(11), 258-264.

Ramírez, C.; Schau, C. y Emmioglu, E. (2012). The Importance of Attitude in Statistics

Education. Statistics Education Research Journal, 11(2), 57-71.

Sandoval, C. (1996). Investigación cualitativa. Bogotá: ARFO.

Skaalvik, S. y Skaalvik, E. (2004). Gender Differences in Math and Verbal Self-Concept, Performance Expectations, and Motivation. Sex Roles, 50(3), 241-252.

Slootmaeckers, K. (2012). Too afraid to learn?! Attitudes towards statistics as a barrier to learning statistics and acquiring quantitative skills. 4th International Conference on Education and New Learning Technologies. Barcelona: Edulearn12.

Sullivan, G. y Artino, A. (2013). Analyzing and Interpreting Data From Likert-Type Scales. Journal of Graduate Medical Education, 5(4), 541-542.

Tejero, C. y Castro, M. (2011). Validación de la escala de actitudes hacia la estadística en estudiantes españoles de ciencias de la actividad física y del deporte. Revista Colombiana de Estadística, 34(1), 1-14.

Universidad Nacional Autónoma de Honduras. (2014). Informe estadístico: resultados de los procesos de admisión del año 2014. Tegucigalpa: Dirección del Sistema de Admisión de la UNAH. 